\title{
Readmission rates after transcatheter aortic valve replacement in high- and extreme-risk patients with severe aortic stenosis
}

\author{
Jessica Forcillo, MD, MSc, ${ }^{\text {,d }}$ Jose F. Condado, MD,${ }^{b}$ Jose N. Binongo, PhD, ${ }^{c}$ Yi Lasanajak, MSPH, \\ Hope Caughron, MD, Vasilis Babaliaros, MD, ${ }^{\mathrm{b}}$ Chandan Devireddy, MD, ${ }^{\mathrm{b}}$ Bradley Leshnower, MD, ${ }^{\mathrm{a}}$ \\ Robert A. Guyton, MD, ${ }^{a}$ Peter C. Block, MD, ${ }^{b}$ Amy Simone, PA-C, ${ }^{\text {a }}$ Patricia Keegan, NP-PhD, \\ Paul Khairy, MD, MSc, ${ }^{d}$ and Vinod H. Thourani, MD $^{\mathrm{a}}$
}

\section{ABSTRACT}

Objective: In high- or extreme-risk patients undergoing transcatheter aortic valve replacement, readmissions have not been adequately studied and are the subject of increased scrutiny by healthcare systems. The objectives of this study were to determine the incidence of 30-day and 1-year cardiac and noncardiac readmissions, identify predictors of readmission, and assess the association between readmission and 1-year mortality.

Methods: A retrospective review was performed on 714 patients who underwent transcatheter aortic valve replacement from September 2007 to January 2015 at Emory University.

Results: Patients' median age was 83 years, and 46.6\% were female. Early allcause readmission for the cohort was $10.5 \%$, and late readmission was $18.8 \%$. Anemia was related to both early all-cause (hazard ratio [HR], 0.74) and cardiovascular-related readmission (HR, 0.60). A 23-mm valve implanted was associated with early all-cause readmission (HR, 1.73). Length of hospital stay was related to late all-cause (HR, 1.14) and cardiovascular-related readmission (HR, 1.21). Postoperative permanent stroke had an impact on late cardiovascular-related readmission (HR, 3.60; 95\% confidence interval, 1.13-11.49). Multivariable analysis identified anemia as being associated with 30-day all-cause readmission, and anemia and postoperative stroke were associated with 30-day cardiovascular-related readmission. Readmissions seemed to be related to 1-year mortality (HR, 2.04; 95\% confidence interval, 1.33-3.12).

Conclusions: We show some baseline comorbidities and procedural complications that are directly associated with early and late readmissions, and anemia and postoperative stroke were associated with an increase in mortality. Moreover, we found that readmission was associated with double the hazard of death within 1 year. Whether treatment of identified risk factors could decrease readmission rates and mortality warrants further investigation. (J Thorac Cardiovasc Surg 2017;154:445-52)

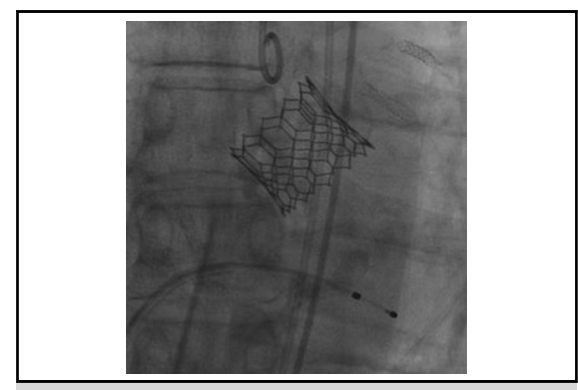

TAVR seen under fluoroscopy after its deployment.

Central Message

Readmission after TAVR is associated with an increased risk of 1-year mortality in a highrisk cohort of patients.

Perspective

In this study, rates of early and late readmission after TAVR in a high-risk cohort of patients were $10.4 \%$ at a median of $19(13-25)$ days and $18.8 \%$ at a median of $129(55-214)$ days, respectively. The majority were not related to a CV cause. Moreover, it was found that readmission was associated with double the hazard of death within 1 year.

See Editorial Commentary page 453.
Transcatheter aortic valve replacement (TAVR) is an alternative to conventional open surgical aortic valve replacement (SAVR) in high-risk patients ${ }^{1-3}$ and is the

From the Divisions of ${ }^{\mathrm{a} C}$ ardiothoracic Surgery and ${ }^{\mathrm{b}}$ Cardiology, Structural Heart and Valve Center, and ${ }^{\mathrm{c}}$ Department of Biostatistics, School of Public Health, Emory University, Atlanta, Ga; and ${ }^{\mathrm{d}}$ Cardiology Department, Université de Montréal, Montréal, Québec, Canada.

Read at the 96th Annual Meeting of The American Association for Thoracic Surgery, Baltimore, Maryland, May 14-18, 2016.

Received for publication May 25, 2016; revisions received March 3, 2017; accepted for publication March 28, 2017; available ahead of print May 19, 2017. procedure of choice in extreme-risk patients (Video 1)., Few reports concerning TAVR and outcomes have evaluated rehospitalization after procedure discharge. ${ }^{6,7}$

\footnotetext{
Address for reprints: Vinod H. Thourani, MD, Emory Hospital Midtown, 550 Peach tree St NE, Medical Office Tower, 6th Floor, Atlanta, GA 30308 (E-mail: vthoura@ emory.edu).

$0022-5223 / \$ 36.00$

Copyright (C) 2017 by The American Association for Thoracic Surgery http://dx.doi.org/10.1016/j.jtcvs.2017.03.144
} 


$\begin{array}{ll}\text { Abbreviations and Acronyms } \\ \text { CI } & =\text { confidence interval } \\ \text { CV } & =\text { cardiovascular } \\ \text { HR } & =\text { hazard ratio } \\ \text { PARTNER } & =\text { Placement of Aortic Transcatheter } \\ & \text { Valve } \\ \text { PROM } & =\text { Predicted Risk of Mortality } \\ \text { SAVR } & =\text { surgical aortic valve replacement } \\ \text { STS } & =\text { Society of Thoracic Surgeons } \\ \text { TAVR } & =\text { transcatheter aortic valve replacement }\end{array}$

Scanning this QR code will take you to a supplemental video. To view the AATS 2016 Webcast, see the URL next to the webcast thumbnail.

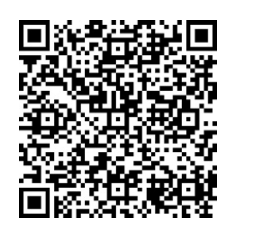

At 1 year, Leon and colleagues ${ }^{4}$ noted a significant decrease in the rate of hospitalization in inoperable patients with TAVR $(22 \%)$ compared with medical therapy $(44 \%)$. When comparing SAVR and TAVR in high-risk patients, Smith and colleagues ${ }^{2}$ noted similar rates of rehospitalization at 1 year (TAVR $18.2 \%$, SAVR $15.5 \%$ ). Although these reports note the overall rates of rehospitalization, studies evaluating the predictors for rehospitalization are uncommon. Readmission is an important health concern, and the rationale for investigation for these predictors remains essential in that they may improve patient outcomes, reduce resource use, and lessen the economic burdens related to TAVR.

The primary objective of this study was to estimate the early (30-day) and late (between 1 month and 1 year) all-cause and cardiovascular (CV)-related readmission rates in high- and extreme-risk patients in a single, high-volume US center. Furthermore, we identified the predictors of all-cause and CV-related readmissions in this cohort of patients. Last, we assessed the association between readmission and 1-year mortality.

\section{MATERIALS AND METHODS}

We retrospectively analyzed 758 patients who underwent TAVR from 2007 to 2015 and are listed in the prospectively entered Emory Society of Thoracic Surgeons (STS) Database. We excluded patients who had died before discharge $(28$ [3.7\%]) and patients who had incomplete follow-up data at 1 year $(16[2.0 \%])$, leaving 714 patients for our analysis (Figure 1). Extracted records from the database included basic demographic information, preexisting comorbidities and other risk factors, and periprocedure and postprocedure clinical outcomes. The study was approved by the Emory University Institutional Review Board in compliance with Health Insurance Portability and Accountability Act regulations and the Declaration of Helsinki. The Institutional Review

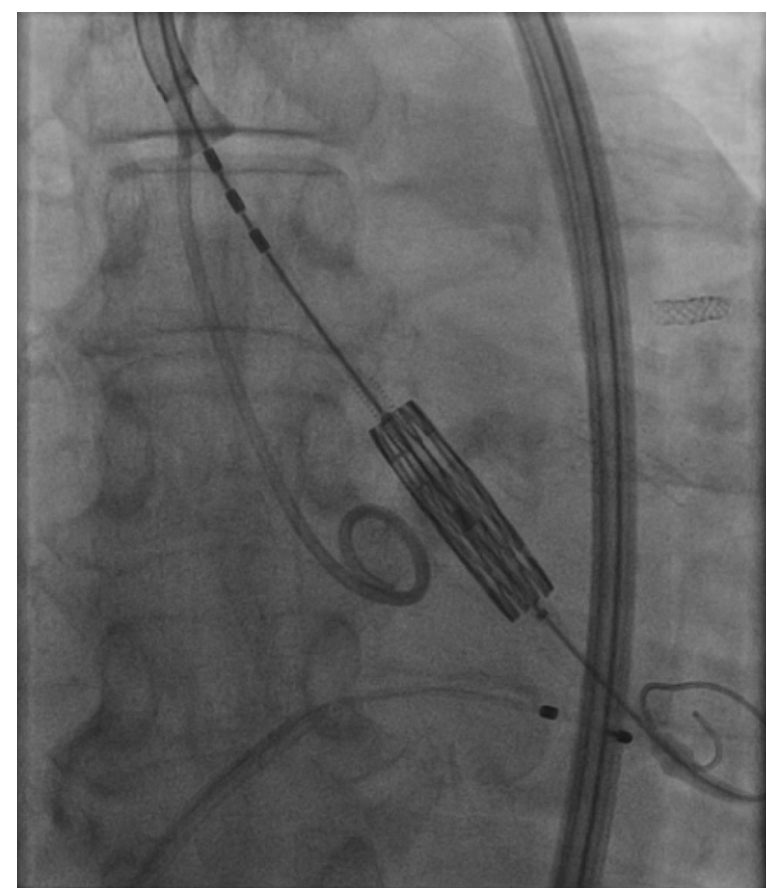

VIDEO 1. Deployment of a TAVR prosthesis. Video available at: http:// www.jtcvsonline.org/article/S0022-5223(17)30705-5/addons.

Board waived individual patient consent because of the retrospective nature of the study.

Patients included in this study were classified as high and extreme risk. High-risk patients were defined as patients with an STS score of more than $8 \%$ with no other comorbidities or anatomic factors that made them inoperable. For extreme-risk patients, we adopted the definition used in the Placement of Aortic Transcatheter Valve (PARTNER) B trial for "inoperable" patients. It includes patients with an STS risk score of $10 \%$ or greater, patients who had coexisting conditions that would be associated with a predicted risk of death of $15 \%$ or greater by 30 days after surgery, or patients who were not considered to be suitable candidates for surgery because they had coexisting conditions that would be associated with a predicted probability of $50 \%$ or more of death by 30 days after surgery or a serious irreversible condition. ${ }^{4}$

Before analysis, preoperative risk factors were identified and extracted from the Emory STS database (which includes 30-day outcome data). Standard STS definitions for risk factors and outcomes were used. Race was dichotomized as Caucasian or non-Caucasian. New York Heart Association heart failure class was dichotomized as class III/IV or I/II. STS discharge location was used to determine where the patients were discharged after their procedure. Valve Academic Research Consortium-2 criteria were used to define major and minor stroke. ${ }^{8}$ Renal failure was defined, according to STS criteria, as an increase in serum creatinine level more than $4.0 \mathrm{mg} / \mathrm{dL}$ or 3 times greater than the baseline creatinine; acute increase must be at least $0.5 \mathrm{md} / \mathrm{dL}$ or a new requirement for dialysis postoperatively. Other clinical outcomes used were defined according to STS criteria. ${ }^{2}$

The access sites for the procedure were transfemoral, transapical, direct transaortic, and other (including transcaval or transcarotid). Patients' charts were reviewed for the subsequent rehospitalizations in any Emory University Hospital. The date, causes of readmission, and discharge location after the hospitalization for readmission were recorded in our database. Follow-up visit or emergency visit notes during the 1-year period after their procedure were reviewed. We also reviewed the notes from our valve nurse navigator or valve coordinator who received phone calls from 


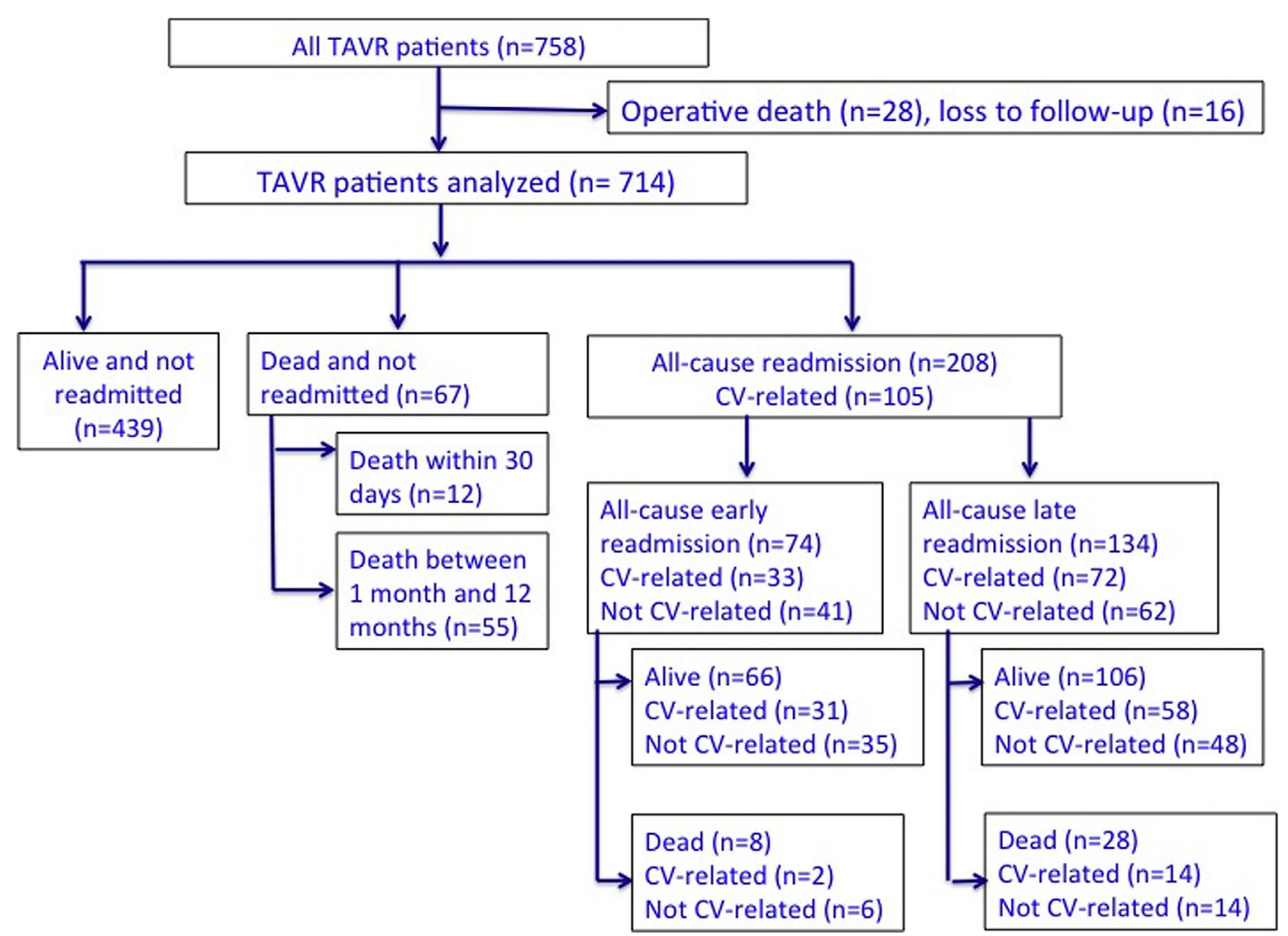

FIGURE 1. Study flow diagram. $T A V R$, Transcatheter aortic valve replacement; $C V$, cardiovascular.

all patients or family when patients were readmitted to any hospitals. Patients were instructed after their index procedure to call them if they experienced any rehospitalizations. The coordinator recorded the readmission status and causes of readmission.
The primary diagnosis on the discharge report of the readmission was used to determine the main cause of readmission after the patient's initial procedure. All-cause readmission included CV readmissions (congestive heart failure, acute coronary syndrome, valve dysfunction, paravalvular leak, aortic

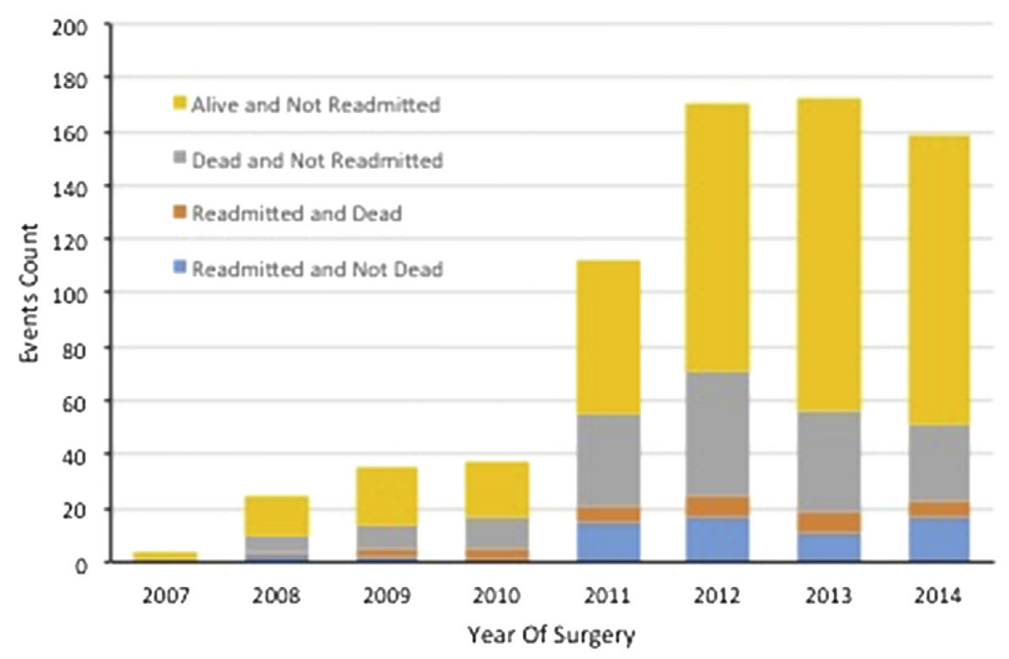

\begin{tabular}{lcccccccc}
\hline Events Type & 2007 & 2008 & 2009 & 2010 & 2011 & 2012 & 2013 & 2014 \\
\hline Readmitted and Not Dead & $25 \%$ & $12 \%$ & $6 \%$ & $3 \%$ & $13 \%$ & $10 \%$ & $6 \%$ & $11 \%$ \\
Readmitted and Dead & $0 \%$ & $4 \%$ & $9 \%$ & $11 \%$ & $5 \%$ & $5 \%$ & $5 \%$ & $4 \%$ \\
Dead and Not Readmitted & $0 \%$ & $24 \%$ & $26 \%$ & $32 \%$ & $30 \%$ & $27 \%$ & $22 \%$ & $18 \%$ \\
Alive and Not Readmitted & $75 \%$ & $60 \%$ & $60 \%$ & $54 \%$ & $51 \%$ & $58 \%$ & $67 \%$ & $68 \%$ \\
\hline Total Counts of Surgery & 4 & 25 & 35 & 37 & 112 & 170 & 172 & 159 \\
\hline
\end{tabular}

FIGURE 2. Trends and events over the study period. 
TABLE 1. Preoperative characteristics

\begin{tabular}{|c|c|c|c|c|c|}
\hline \multirow[b]{2}{*}{ Variables } & \multirow[b]{2}{*}{$\begin{array}{c}\text { Median (IQR) } \\
\text { or count }(\%) \\
(n=714)\end{array}$} & \multicolumn{2}{|c|}{ All-cause readmission } & \multicolumn{2}{|c|}{ CV-related readmission } \\
\hline & & $\begin{array}{c}\text { HR }(95 \% \mathrm{CI}) \\
\text { Early readmission } \\
\quad(\text { within } 30 \mathrm{~d})\end{array}$ & $\begin{array}{c}\text { HR }(95 \% \text { CI }) \\
\text { Late readmission } \\
\text { (from } 1 \text { mo to } 1 \mathrm{y})\end{array}$ & $\begin{array}{c}\text { HR }(95 \% \mathrm{CI}) \\
\text { Early readmission } \\
\quad(\text { within } 30 \mathrm{~d})\end{array}$ & $\begin{array}{c}\text { HR }(95 \% \text { CI }) \\
\text { Late readmission } \\
\text { (from } 1 \text { mo to } 1 \mathrm{y})\end{array}$ \\
\hline Age (y) & $83(77-87)$ & $0.99(0.82-1.20)$ & $1.19(0.98-1.43)$ & $1.01(0.74-1.38)$ & $1.03(0.81-1.32)$ \\
\hline Female sex & $333(46.6)$ & $1.35(0.85-2.167)$ & $1.11(0.8-1.54)$ & $1.49(0.81-2.76)$ & $1.06(0.65-1.72)$ \\
\hline BMI & $25.9(22.7-29.9)$ & $1.01(0.77-1.34)$ & $0.98(0.81-1.17)$ & $1.04(0.76-1.41)$ & $0.84(0.64-1.11)$ \\
\hline Diabetes & $311(43.6)$ & $1.16(0.72-1.85)$ & $1.00(0.72-1.39)$ & $1.11(0.6-2.05)$ & $0.89(0.54-1.47)$ \\
\hline Hypertension & $681(95.4)$ & $0.73(0.26-2.07)$ & $3.61(0.89-14.71)$ & $0.87(0.21-3.58)$ & $1.60(0.39-6.53)$ \\
\hline Moderate/severe COPD & $220(30.8)$ & $1.19(0.73-1.94)$ & $0.69(0.47-1.00)$ & $1.29(0.68-2.44)$ & $0.54(0.29-0.98)$ \\
\hline NYHA (class III/IV) & $651(91.8)$ & $0.80(0.37-1.72)$ & $0.78(0.44-1.38)$ & $1.15(0.36-3.73)$ & $0.64(0.30-1.34)$ \\
\hline $\mathrm{CAD}$ (prior $\mathrm{MI}, \mathrm{CABG}$, or $\mathrm{PCI}$ ) & $438(61.3)$ & $1.26(0.76-2.08)$ & $0.83(0.6-1.16)$ & $1.09(0.58-2.06)$ & $0.83(0.51-1.36)$ \\
\hline Prior PCI & 247 (34.6) & $1.47(0.91-2.35)$ & $0.71(0.49-1.02)$ & $1.51(0.81-2.79)$ & $1.07(0.64-1.78)$ \\
\hline Previous cardiac surgery & $336(47.1)$ & $0.82(0.51-1.32)$ & $0.83(0.597-1.167)$ & $0.80(0.429-1.486)$ & $0.88(0.539-1.448)$ \\
\hline Previous AV surgery & $230(32.2)$ & $1.37(0.85-2.22)$ & $1.16(0.82-1.65)$ & $1.37(0.73-2.57)$ & $1.53(0.93-2.54)$ \\
\hline Prior CV disease & $224(31.4)$ & $1.10(0.67-1.81)$ & $1.02(0.71-1.47)$ & $1.02(0.53-1.97)$ & $0.85(0.49-1.48)$ \\
\hline Dialysis & $25(3.5)$ & $1.79(0.67-4.79)$ & $1.31(0.52-3.26)$ & $2.26(0.7-7.32)$ & $1.13(0.28-4.64)$ \\
\hline Liver disease & $27(3.8)$ & $1.21(0.39-3.81)$ & $1.65(0.77-3.51)$ & $1.35(0.33-5.58)$ & $1.42(0.45-4.54)$ \\
\hline PAD & $241(33.8)$ & $1.24(0.77-2.01)$ & $0.79(0.55-1.14)$ & $1.52(0.82-2.82)$ & $1.21(0.73-2)$ \\
\hline Immunocompromised & $101(14.2)$ & $1.32(0.71-2.44)$ & $1.16(0.75-1.79)$ & $1.52(0.7-3.3)$ & $1.04(0.51-2.1)$ \\
\hline STS PROM & $10.04(7.2-13.9)$ & $1.11(0.93-1.33)$ & $0.92(0.78-1.08)$ & $1.19(0.95-1.5)$ & $0.87(0.66-1.16)$ \\
\hline $\mathrm{EF} \leq 35 \%$ & 147 (20.6) & $0.86(0.48-1.57)$ & $1.10(0.74-1.64)$ & $0.90(0.41-1.94)$ & $1.50(0.87-2.58)$ \\
\hline Moderate/severe MR & 337 (47.2) & $1.22(0.76-1.95)$ & $1.02(0.73-1.43)$ & $1.42(0.77-2.63)$ & $1.48(0.9-2.42)$ \\
\hline Hemoglobin $(\mathrm{g} / \mathrm{dL})$ & $11.4(10.2-12.6)$ & $0.74(0.59-0.93)^{*}$ & $0.88(0.74-1.04)$ & $0.60(0.44-0.81)^{*}$ & $0.84(0.65-1.09)$ \\
\hline
\end{tabular}

$I Q R$, Interquartile range; $H R$, hazard ratio; $C I$, confidence interval; $B M I$, body mass index; $C O P D$, chronic obstructive pulmonary disease; $N Y H A$, New York Heart Association; $C A D$, coronary artery disease; $M I$, myocardial infarction; $C A B G$, coronary artery bypass graft; $P C I$, percutaneous intervention; $A V$, aortic valve; $C V$, cardiovascular; $P A D$, peripheral artery disease; STS PROM, Society of Thoracic Surgeons Predicted Risk of Mortality; $E F$, ejection fraction; $M R$, mitral regurgitation. *P less than .05.

stenosis, aortic insufficiency, endocarditis, and thrombus), arrhythmia/heart block, stroke, and vascular complications. Non-CV causes were divided into infection (genitourinary, gastrointestinal, and bacteremia without endocarditis), respiratory (pneumonia, bronchitis, pleural effusions, chronic obstructive pulmonary disease), gastrointestinal bleeding and bleeding related to anticoagulation regimen, trauma (falls, fractures), renal failure, and other (other noncardiac surgeries or endocrine, debilitation, and psychiatric). Time to readmission was calculated as the time between the date of hospital discharge after the index TAVR procedure and the first hospital readmission day. The discharge location from the index operation was collected using our Emory STS database and confirmed with the chart's review.

\section{Statistical Analysis}

Preoperative, operative, and postoperative characteristics are summarized as counts (proportions) or mean (standard deviation); skewed continuous data are summarized as median (interquartile range, Q1-Q3). Only first readmission cases were considered in the analysis. Patients who died during the operation and without 1-year follow-up were excluded from the analysis. There were 3 primary questions: (1) What was the all-cause and CV-related readmission rate? (2) How is each preoperative, operative, and postoperative variable related to readmission (both all-cause and CV-related)? (3) What is the association between readmission and death? In question 2, death is a competing risk, because death precludes readmission. Because the Kaplan-Meier method is inappropriate, we resorted to applying Fine and Gray's ${ }^{9}$ extension of Cox regression that models the hazards of the cumulative incidence function. To estimate the hazard ratio (HR) for early and late readmission, we introduced a heaviside function to divide follow-up time into less than 1 month and between 1 month and 1 year. We then estimated separate HRs for each time interval. In the multivariable analysis, age, sex, race, left ventricular ejection fraction, body mass index, moderate or severe chronic obstructive pulmonary disease, coronary artery disease, previous cardiac surgery, previous percutaneous intervention, previous CV disease, dialysis, liver disease, preoperative hemoglobin, valve size, and length of stay were incorporated in the model for all-cause readmission; preoperative hemoglobin, length of stay, procedure time, and postoperative stroke were included in the model for CV-related readmission. In question 3, we treated readmission as a time-varying variable in the Cox regression model, with death as the event of interest. ${ }^{9}$ All tests of hypotheses were 2 sided and performed at the .05 level of significance. SAS 9.4 (SAS Institute Inc, Cary, $\mathrm{NC}$ ) was used for all the data analyses.

\section{RESULTS}

Figure 2 illustrates the trends over the year for readmission. Except for the first year when 4 procedures were effectuated, the rate of readmission remains fairly constant between $6 \%$ and $12 \%$ (Figure 2).

\section{Predictors for Readmission}

Preoperative characteristics. Table 1 lists the preoperative characteristics and shows the unadjusted HRs for all-cause and CV-related early (30-day) and late (from 1 month to 1 year) readmission. The median age was 83 years (interquartile range, $77-87)$, and 333 patients $(46.6 \%)$ were female. Most patients were high risk with a median STS Predicted Risk of Mortality (PROM) score of $10.0 \%$ (interquartile range, 7.2-13.9). Preoperative hemoglobin was a preoperative characteristic associated with early all-cause and CV-related readmission (HR, 0.74; $95 \%$ confidence 


\begin{tabular}{|c|c|c|c|c|c|}
\hline \multirow[b]{2}{*}{ Variables } & \multirow[b]{2}{*}{$\begin{array}{c}\text { Median (IQR) } \\
\text { or count }(\%) \\
(n=714)\end{array}$} & \multicolumn{2}{|c|}{ All-cause readmission } & \multicolumn{2}{|c|}{ CV-related readmission } \\
\hline & & $\begin{array}{c}\text { HR }(95 \% \text { CI }) \\
\text { Early } \\
\text { readmission } \\
(\text { within } 30 \mathrm{~d})\end{array}$ & $\begin{array}{c}\text { HR }(95 \% \text { CI }) \\
\text { Late } \\
\text { readmission } \\
\text { (from } 1 \text { mo to } 1 \text { y) }\end{array}$ & $\begin{array}{c}\text { HR }(95 \% \text { CI }) \\
\text { Early readmission } \\
\quad(\text { within } 30 \mathrm{~d})\end{array}$ & $\begin{array}{c}\text { HR }(95 \% \text { CI }) \\
\text { Late readmission } \\
(\text { from } 1 \text { mo to } 1 \mathrm{y})\end{array}$ \\
\hline Need for intra-aortic balloon pump & $20(2.8)$ & $0.50(0.07-3.45)$ & $2.22(1.02-4.83)$ & $0.84(0.12-6.13)$ & $2.63(0.95-7.25)$ \\
\hline \multicolumn{6}{|l|}{ Valve type } \\
\hline SAPIEN (Edwards Lifesciences, Irvine, Calif) & $458(64.6)$ & $0.96(0.59-1.56)$ & $1.06(0.75-1.52)$ & $0.69(0.37-1.29)$ & $1.47(0.83-2.59)$ \\
\hline SAPIEN XT & $251(35.4)$ & $1.05(0.64-1.71)$ & $0.94(0.66-1.34)$ & $1.44(0.78-2.67)$ & $0.68(0.39-1.2)$ \\
\hline \multicolumn{6}{|l|}{ Valve size } \\
\hline $23 \mathrm{~mm}(23 \mathrm{~mm}$ vs $26+29 \mathrm{~mm})$ & $332(46.5)$ & $1.64(1.02-2.63)^{*}$ & $1.17(0.84-1.62)$ & $1.24(0.67-2.29)$ & $1.37(0.84-2.23)$ \\
\hline $26 \mathrm{~mm}(26 \mathrm{~mm}$ vs $23+29 \mathrm{~mm})$ & $307(43.0)$ & $0.61(0.37-1.01)$ & $0.88(0.63-1.23)$ & $0.75(0.4-1.41)$ & $0.80(0.48-1.32)$ \\
\hline $29 \mathrm{~mm}(29 \mathrm{~mm}$ vs $23+26 \mathrm{~mm})$ & $70(9.8)$ & $1.02(0.47-2.2)$ & $0.96(0.52-1.77)$ & $1.27(0.5-3.23)$ & $0.67(0.24-1.83)$ \\
\hline Access (transfemoral) (transfemoral vs other) & $420(58.8)$ & $1.03(0.64-1.67)$ & $1.01(0.72-1.42)$ & $0.99(0.53-1.85)$ & $0.83(0.51-1.36)$ \\
\hline \multicolumn{6}{|l|}{ Alternative access } \\
\hline TA & $199(27.9)$ & $0.70(0.4-1.25)$ & $0.99(0.68-1.44)$ & $0.82(0.4-1.67)$ & $1.02(0.59-1.75)$ \\
\hline TAo & $69(9.7)$ & $1.65(0.85-3.2)$ & $0.92(0.51-1.67)$ & $1.68(0.71-4)$ & $1.24(0.57-2.73)$ \\
\hline Minimalist TAVR & $200(28)$ & $1.00(0.58-1.67)$ & $0.82(0.56-1.2)$ & $1.35(0.71-2.58)$ & $0.99(0.58-1.71)$ \\
\hline Second valve implanted & $44(6.2)$ & $0.96(0.34-2.62)$ & $1.09(0.55-2.13)$ & $1.69(0.6-4.73)$ & $1.67(0.72-3.88)$ \\
\hline Need for postoperative balloon dilatation & $195(27.3)$ & $1.17(0.7-1.94)$ & $0.87(0.58-1.29)$ & $1.54(0.81-2.9)$ & $0.95(0.54-1.68)$ \\
\hline Procedural time (min) & $114(95-142)$ & $0.96(0.78-1.19)$ & $1.14(0.97-1.34)$ & $0.83(0.58-1.2)$ & $1.27(1.05-1.52)^{*}$ \\
\hline Fluoroscopy time & $22.2(17.0-29.2)$ & $1.12(0.91-1.38)$ & $1.16(0.99-1.37)$ & $1.19(0.92-1.55)$ & $0.88(0.54-1.43)$ \\
\hline Contrast (mL) & $110(75-160)$ & $0.77(0.55-1.07)$ & $0.91(0.68-1.22)$ & $0.80(0.51-1.25)$ & $0.83(0.54-1.27)$ \\
\hline Procedure success & $599(83.9)$ & $1.13(0.58-2.21)$ & $1.06(0.67-1.66)$ & $1.11(0.47-2.65)$ & $0.77(0.42-1.42)$ \\
\hline \multicolumn{6}{|l|}{ Discharge PVL } \\
\hline None/mild & $686(96.1)$ & $0.72(0.18-2.9)$ & $0.77(0.32-1.85)$ & $0.62(0.09-4.54)$ & $0.75(0.18-3.05)$ \\
\hline Moderate/severe & $28(3.9)$ & & & & \\
\hline Discharge mean gradient & $10.1(8.0-14.0)$ & $1.06(0.89-1.27)$ & $1.00(0.84-1.19)$ & $1.07(0.8-1.44)$ & $1.03(0.8-1.33)$ \\
\hline Postprocedure LVEDP & $20(18.0-27.0)$ & $1.04(0.78-1.37)$ & $1.11(0.9-1.38)$ & $0.91(0.59-1.4)$ & $1.09(0.79-1.51)$ \\
\hline Blood transfusion in operating room & $140(19.6)$ & $0.84(0.45-1.56)$ & $1.24(0.84-1.85)$ & $0.68(0.29-1.62)$ & $1.31(0.74-2.31)$ \\
\hline Units of red blood cells transfused & $1.5(1.0-2.0)$ & $0.51(0.24-1.09)$ & $1.16(0.81-1.59)$ & $0.79(0.29-2.19)$ & $1.22(0.82-1.8)$ \\
\hline Discharge location nursing facility & $11(1.5)$ & - & $0.77(0.19-3.09)$ & - & $2.11(0.52-8.63)$ \\
\hline
\end{tabular}

$I Q R$, Interquartile; $C V$, cardiovascular; $H R$, hazard ratio; $C I$, confidence interval; $T A$, transapical; $T A o$, direct transaortic; $T A V R$, transcatheter aortic valve replacement; $P V L$, paravalvular leak; $L V E D P$, left ventricular end-diastolic pressure. ${ }^{*} P$ value less than .05 .

interval [CI], 0.59-0.93 and HR, 0.60, 95\% CI, 0.44-0.81, respectively).

Operative characteristics. Table 2 displays the operative characteristics and the unadjusted HRs for early and late all-cause and CV-related readmission. Implantation of a 23-mm valve was related to 30-day all-cause readmission (HR, 1.73; 95\% CI, 1.0-32-2.90). A longer procedural time was a significant predictor for late $\mathrm{CV}$ readmission (HR, 1.27; 95\% CI, 1.05-1.52).

Postoperative characteristics. There were no statistically significant postoperative parameters associated with early all-cause readmission (Table 3). However, the length of hospital stay (HR, 1.14; 95\% CI, 1.01-1.29) was associated with a higher late all-cause and CV-related readmission (HR, 1.21; 95\% CI, 1.03-11.43). Of note, postoperative paravalvular leak was not associated with early or late readmission (HR, 0.53; 95\% CI, 0.17-1.67, HR, 1.32; 95\% CI, 0.75-2.31, respectively). Postoperative permanent stroke (HR, 3.60; 95\% CI, 1.13-11.49) was associated with late $\mathrm{CV}$-related readmission.
Multivariable analysis showed that anemia (adjusted HR, $0.78 ; 95 \%$ CI, 0.62-0.99) was significantly associated with early all-cause readmission. Anemia (adjusted HR, 0.61; 95\% CI, 0.44-0.84) and postoperative stroke (adjusted HR, 5.98; 95\% CI, 1.25-28.54) were both significantly associated with early CV-related readmission (Table 4).

\section{Reasons for Readmission}

The reasons for early and late all-cause readmission are shown in Table 4. At 30 days, 74 patients $(10.4 \%)$ had all-cause readmission to a hospital at a median of 19 (13-25) days, in whom the majority (41 patients, 56.8\%) did not have a CV cause. Of those 33 patients $(44.6 \%)$ who were admitted related to $\mathrm{CV}$ disease, the most common was congestive heart failure $(57.6 \%)$ at a median time of 20 (11-25) days. Between the 30-day and 1-year period, 134 patients $(18.8 \%)$ were readmitted to a hospital at a median of 129 (55-214) days; this was not related to a CV cause in the majority (62 patients, $46.3 \%)$. Of those $72(53.7 \%$ ) who 
TABLE 3. Postoperative characteristics

\begin{tabular}{|c|c|c|c|c|c|}
\hline \multirow[b]{2}{*}{ Variables } & \multirow[b]{2}{*}{$\begin{array}{c}\text { Median }(I Q R) \\
\text { or count }(\%) \\
(n=714) \\
\end{array}$} & \multicolumn{2}{|c|}{ All-cause readmission } & \multicolumn{2}{|c|}{ CV-related readmission } \\
\hline & & $\begin{array}{c}\text { HR }(95 \% \mathrm{CI}) \\
\text { Early } \\
\text { readmission } \\
(\text { within } 30 \mathrm{~d}) \\
\end{array}$ & $\begin{array}{c}\text { HR }(95 \% \text { CI }) \\
\text { Late } \\
\text { readmission } \\
\text { (from } 1 \text { mo to } 1 \text { y) }\end{array}$ & $\begin{array}{c}\text { HR }(95 \% \mathrm{CI}) \\
\text { Early readmission } \\
\quad(\text { within } 30 \mathrm{~d})\end{array}$ & $\begin{array}{c}\text { HR }(95 \% \text { CI }) \\
\text { Late readmission } \\
\text { (from } 1 \text { mo to } 1 \text { y) }\end{array}$ \\
\hline Length of ICU stay (for HR, 5-h increment)* & $29(22.5-55.3)$ & $0.99(0.98-1.00)$ & $1.01(1.00-1.01)$ & $0.99(0.96-1.01)$ & $1.01(1.00-1.02)$ \\
\hline $\begin{array}{l}\text { Length of hospital stay (for HR, } \\
1 \mathrm{SD}=6.11 \mathrm{~d} \text { increment)* }\end{array}$ & $65(3.0-7.0)$ & $0.92(0.78-1.08)$ & $1.14(1.01-1.29)^{*}$ & $0.86(0.58-1.26)$ & $1.21(1.03-1.43)^{*}$ \\
\hline Major vascular complication & $15(2.1)$ & - & $1.48(0.52-4.16)$ & - & $2.53(0.79-8.08)$ \\
\hline Bleeding by severity (major/life-threatening) & $111(7.6)$ & $1.40(0.78-2.5)$ & $1.24(0.80-1.91)$ & $1.34(0.62-2.91)$ & $0.89(0.42-1.86)$ \\
\hline Postoperative permanent stroke & $13(1.8)$ & $1.63(0.40-6.64)$ & $2.31(0.98-5.47)$ & $3.02(0.73-12.50)$ & $3.60(1.13-11.49)^{*}$ \\
\hline Postoperative atrial fibrillation & $58(8.1)$ & $0.90(0.36-2.24)$ & $0.59(0.28-1.25)$ & $0.94(0.29-3.05)$ & $0.39(0.10-1.61)$ \\
\hline Postoperative cardiac arrest & $11(1.5)$ & $1.87(0.49-7.09)$ & $1.06(0.26-4.3)$ & $3.16(0.76-13.08)$ & - \\
\hline Pacemaker need & $33(4.6)$ & $1.30(0.49-3.46)$ & $0.57(0.21-1.6)$ & $1.14(0.28-4.71)$ & $1.01(0.32-3.21)$ \\
\hline Postoperative prolonged ventilation & $63(8.8)$ & $0.64(0.24-1.75)$ & $1.04(0.57-1.88)$ & $0.57(0.14-2.34)$ & $1.33(0.57-3.09)$ \\
\hline Postoperative renal failure & $13(1.8)$ & $0.79(0.1-6.1)$ & $1.49(0.53-4.19)$ & - & $2.83(0.89-9.03)$ \\
\hline Postoperative dialysis required & $8(1.1)$ & $1.36(0.17-10.94)$ & $0.53(0.07-4)$ & - & - \\
\hline $\mathrm{EF} 30-\mathrm{d}$ (for $\mathrm{HR}, 1 \mathrm{SD}=1.37$ increment) $*$ & $5(4.0-6.0)$ & $1.33(0.97-1.81)$ & $0.96(0.77-1.2)$ & $1.30(0.87-1.95)$ & $0.84(0.64-1.10)$ \\
\hline Postmitral regurgitation & $135(18.9)$ & $1.54(0.91-2.62)$ & $0.86(0.54-1.38)$ & $1.58(0.79-3.16)$ & $1.23(0.67-2.27)$ \\
\hline $\begin{array}{l}\text { Postaortic valve mean gradient } 30 \mathrm{~d} \\
\text { (for } \mathrm{HR}, 1 \mathrm{SD}=4.79 \text { increment)* }\end{array}$ & $9.5(7.0-12.5)$ & $1.08(0.93-1.26)$ & $0.93(0.77-1.12)$ & $1.04(0.77-1.40)$ & $0.91(0.70-1.20)$ \\
\hline PVL 30 d echo (moderate/severe) & $54(7.6)$ & $0.53(0.17-1.67)$ & $1.32(0.75-2.31)$ & - & $1.57(0.72-3.44)$ \\
\hline
\end{tabular}

$I Q R$, Interquartile range; $C V$, cardiovascular; $H R$, hazard ratio; $C I$, confidence interval; $I C U$, intensive care unit; $E F$, ejection fraction; $S D$, standard deviation; $P V L$, paravalvular leak. *For continuous variables, HR compares the hazard between 2 groups that differ by the increment specified.

were admitted for $\mathrm{CV}$ disease, the most common cause was congestive heart failure $(48.6 \%)$ and the median time was 64 days (35-161).

\section{Effect of Readmission on Survival}

A total of 439 patients $(61.5 \%)$ did not have any readmission after TAVR, whereas 142 patients $(19.9 \%)$ had 1 readmission and 66 patients $(9.2 \%)$ had 2 or more

TABLE 4. Rate and reasons for readmission

\begin{tabular}{lcc}
\hline & $\begin{array}{c}\text { Early readmission } \\
\text { (within } \mathbf{3 0} \text { d) } \\
(\mathbf{N}=\mathbf{7 4})\end{array}$ & $\begin{array}{c}\text { Late readmission } \\
\text { (from 1 mo to 1 y) } \\
(\mathbf{N}=\mathbf{1 3 4})\end{array}$ \\
\hline $\mathrm{CV}$ & Total: $\mathrm{n}=33$ & Total: $\mathrm{n}=72$ \\
Congestive heart failure & 19 & 35 \\
Arrhythmia/heart block & 5 & 10 \\
Vascular complications & 5 & 8 \\
Acute coronary syndrome & 2 & 8 \\
Valve dysfunction/PVL leak & 1 & 3 \\
Stroke/TIA & 0 & 8 \\
Non-CV \% & Total: $\mathrm{n}=41$ & Total: $\mathrm{n}=62$ \\
Other* & 24 & 24 \\
Respiratory & 7 & 12 \\
GI or ACO-related bleeding & 3 & 10 \\
Infection & 3 & 10 \\
Traumatology & 3 & 3 \\
Renal failure & 2 & 3 \\
\hline
\end{tabular}

$C V$, Cardiovascular; $P V L$, paravalvular leak; $T I A$, transient ischemic attack; $G I$, gastrointestinal; $A C O$, anticoagulation. *Other includes surgical abdominal-urinary procedures, psychiatric disorders, and debilitation. readmissions. Of those who were readmitted, $36(17 \%)$ died during readmission. The mortality comparison between readmission and no readmission is complicated by the fact that there were 67 patients who died without being readmitted. By treating readmission appropriately as time-dependent, we found that being readmitted doubles the (unadjusted) hazard of death at 1 year (HR, 2.04; 95\% CI, 1.33-3.12).

\section{DISCUSSION}

The majority of studies evaluating TAVR have concentrated on outcomes associated with the morbidity and mortality of this transformative procedure and less on the rates and causes of readmissions. In the current study, we have expanded on the impact of readmissions after TAVR for severe aortic stenosis in high- and extreme-risk patients. The early all-cause readmission is $10.4 \%$, and late readmission is $18.8 \%$. The rate of early $\mathrm{CV}$ readmission was $4.6 \%$ and $10.1 \%$ between 30 days and 1 year, most likely occurring from congestive heart failure.

Previous studies have shown that advanced age, male sex, end-stage renal disease, severe chronic obstructive pulmonary disease, nontransfemoral access, and preoperative atrial fibrillation can predict 1-year mortality in patients undergoing TAVR..$^{10}$ In this study, anemia, hospital length of stay, and postoperative stroke were predictors of readmission, and readmission was a predictor of 1-year mortality. It is possible that a constellation of risk factors not included in the STS score are predictive of more readmissions. In this 
study, we used the STS PROM as a continuous variable and do not have distinct recommendations for a cutoff value. Makkar and colleagues ${ }^{11}$ have shown that in those extreme-risk patients with an STS PROM of $15 \%$ or greater, TAVR had a similar survival as medical therapy. ${ }^{11}$ However, we did not find that a higher STS PROM score was predictive of higher 1-year all-cause and CV readmissions. Our data are supported by a report from Rodes-Cabau and colleagues, ${ }^{12}$ who also showed an association between readmissions and life-threatening bleeding and lower hemoglobin. Transfemoral versus alternative access approaches and the degree of postoperative paravalvular leaks were not associated with an increased 30 -day or 1-year readmission rate. We included those parameters because they have been associated in previous studies with increased mortality, ${ }^{1,2}$ but in fact they had no impact on readmissions. Also in our study, we found that the 23-mm valve size was associated with a greater number of early readmissions than the 26- and 29-mm valve sizes. We can speculate, but we do not know for sure, that the $23-\mathrm{mm}$ valve was associated with more readmissions because of its smaller effective orifice area that may lead to prosthesis-patient mismatch, which results in more early and late clinical events due to decrease left ventricular mass regression and decreased improvement of clinical symptoms. A smaller prosthesis sometimes needs to be implanted because of anatomic concerns, such as important calcifications of the sinotubular junction or the annulus, reflecting sicker patients who could be readmitted more and resulting in imperfect results causing perivalvular or central leaks that could lead to an increase in mortality. ${ }^{13}$

One of the advantages of TAVR has been the limited invasiveness to the patient compared with surgical valve replacement. Awad and colleagues ${ }^{14}$ showed that although patients undergoing TAVR were older and sicker, there were no CV-related readmissions within 30 days for TAVR, and the time to first readmission was significantly longer than for patients undergoing SAVR. ${ }^{14}$ The rate of CV readmission for the patients undergoing SAVR aged more than 80 years was $13 \%$ with a mean time for first readmission at 9.7 days. The PARTNER 1A trial, one of the largest studies that compared TAVR with SAVR in a highrisk cohort of patients, showed that the rate of 30-day repeat hospitalization for cardiac reasons was $4.4 \%$ in the TAVR group versus $3.7 \%$ in the surgical replacement group $(P=$ not significant $){ }^{2}$ At 1 year, this difference was similar with a CV readmission rate of $18.2 \%$ in the TAVR group and $15.5 \%$ in SAVR. The rate of CV readmission in the current study was closely similar to the PARTNER study at 30 days and 1 year at $4.6 \%$ and $11.1 \%$, respectively. A recent propensity-matched study comparing readmission rates in 389 TAVR with 389 SAVR cases by Hannan and colleagues ${ }^{15}$ showed that the 30-day all-cause readmission rate was similar between groups at 30 days (TAVR 18.8\% vs SAVR $19.3 \%, P=.86) .{ }^{15}$ For patients aged at least 80 years, they showed a 30-day all-cause readmission of $19.9 \%$ and $22 \%$, respectively $(P=.59)$. In this analysis, they noticed more all-cause readmissions after SAVR at 4 and 10 days after discharge, whereas more readmissions after TAVR occurred 11 and 20 days after discharge. ${ }^{15}$ In the current study, the median time was 19 days for allcause 30-day readmission and 20 days for $\mathrm{CV}$ readmission.

The impact on the correlation between readmission rates and the impact on societal resource use remain undefined. The subsequent readmission burden and the cost associated with this should be further evaluated in a cost-benefit analysis study to better understand the impact of this innovative procedure in high-risk patients. It may be reasonable to include not only cardiologists and cardiac surgeons in our heart teams but also other nursing and social services professionals in ascertaining those at high risk for readmission. This is important for the allocation of this innovative procedure and the cost to the society.

At Emory, we have made a paradigm shift in how we perform transfemoral TAVR with the use of the minimalist technique, which is not completely shown in the current study because we started this study in 2007 and started using the minimalist technique in 2012. Minimalist TAVR has been associated with less intensive care unit and hospital stays with almost no need for intubation, all of which have been shown to increase readmission rates. ${ }^{15} \mathrm{We}$ are also now aggressive in treating and finding the sources of a low preoperative anemia, which may include preprocedural workup by gastroenterology and hematology. We continue to have robust heart team discussions on those patients with a high STS score, with moderate to severe mitral regurgitation, and who need valve-in-valve procedures that may leave the patient with moderate aortic stenosis. We also have implemented a nurse navigator who sees patients during their preadmission visit and follows them closely regarding vital signs, diet, and medications to prevent readmission for congestive heart failure.

\section{Study Limitations}

The study is retrospective, which makes it vulnerable to selection bias. Moreover, this study was performed in a single-center, high-volume, and experienced institution, and the results may not be generalized to other institutions. There is also potential recall bias for readmission when the patients were interviewed during their clinic appointments. Also, we did not evaluate readmission as a recurring event. It is likely that the health care burden of readmission in this current study is underestimated. Another limitation is related to the use of cumulative incidence function analysis for competing risks data: Patients who die before the typical follow-up might have less valid readmission data because of the type of follow-up. As such, we may be underestimating readmissions in patients with early 1-year mortality. 


\section{CONCLUSIONS}

We show some baseline comorbidities and procedural complications that are directly associated with early and late readmissions, and anemia and postoperative stroke were associated with an increased in mortality. Moreover, we found that readmissions doubled the hazard of death within 1 year. Whether treatment of identified risk factors could decrease readmission rates and mortality warrants further investigation.

\section{Webcast}

You can watch a Webcast of this AATS meeting presentation by going to: http://webcast.aats.org/2016/Video/Wednesday/ 05-18-16_Ballroom_I_0918_Forcillo-800.mp4.

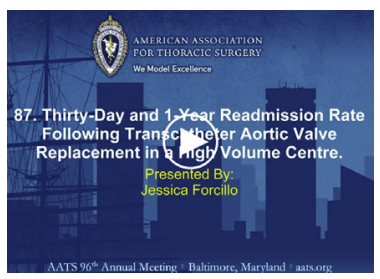

\section{Conflict of Interest Statement}

Authors have nothing to disclose with regard to commercial support.

\section{References}

1. Mack MJ, Leon MB, Smith CR, Miller DC, Moses JW, Tuzcu EM, et al; PARTNER 1 trial investigators. 5-year outcomes of transcatheter aortic valve replacement or surgical aortic valve replacement for high surgical risk patients with aortic stenosis (PARTNER 1): a randomised controlled trial. Lancet. 2015;385:2477-84.

2. Smith CR, Leon MB, Mack MJ, Miller DC, Moses JW, Svensson LG, et al. Transcatheter versus surgical aortic-valve replacement in high-risk patients. N Engl J Med. 2011;364:2187-98.

3. Adams DH, Popma JJ, Reardon MJ, Yakubov SJ, Coselli JS, Deeb GM, et al; U.S CoreValve Clinical Investigators. Transcatheter aortic-valve replacement with a self-expanding prosthesis. N Engl J Med. 2014;370:1790-8.
4. Leon MB, Smith CR, Mack M, Miller DC, Moses JW, Svensson LG, et al. Transcatheter aortic-valve implantation for aortic stenosis in patients who cannot undergo surgery. N Engl J Med. 2010;363:1597-607.

5. Kapadia SR, Leon MB, Makkar RR, Tuzcu EM, Svensson LG, Kodali S, et al; PARTNER trial investigators. 5-year outcomes of transcatheter aortic valve replacement compared with standard treatment for patients with inoperable aortic stenosis (PARTNER 1): a randomised controlled trial. Lancet. 2015;385: 2485-91.

6. Leon MB, Smith CR, Mack MJ, Makkar RR, Svensson LG, Kodali SK, et al; PARTNER trial investigators. Transcatheter or surgical aortic-valve replacement in intermediate-risk patients. $N$ Engl J Med. 2016;374: 1609-20.

7. Thourani VH, Kodali S, Makkar RR, Herrmann HC, Williams M, Babaliaros V, et al. Transcatheter aortic valve replacement versus surgical valve replacement in intermediate-risk patients: a propensity score analysis. Lancet. 2016;387: 2218-25.

8. Kappetein AP, Head SJ, Genereux P, Piazza N, van Mieghem NM, Blackstone EH, et al. Valve Academic Research Consortium-2. Updated standardized endpoint definitions for transcatheter aortic valve implantation: the Valve Academic Research Consortium-2 consensus document (VARC-2). Eur J Cardiothorac Surg. 2012;42:S45-60.

9. Fine JP, Gray RJ. A proportional hazards model for the subdistribution of a competing risk. J Am Stat Assoc. 1999;94:496-509.

10. Holmes DR Jr, Brennan JM, Rumsfeld JS, Dai D, O'Brien SM, Vemulapalli S, et al; STS/ACC TVT Registry. Clinical outcomes at 1 year following transcatheter aortic valve replacement. JAMA. 2015;313:1019-28.

11. Makkar RR, Fontana GP, Jilaihawi H, Kapadia S, Pichard AD, Douglas PS, et al. Transcatheter aortic-valve replacement for inoperable severe aortic stenosis. N Engl J Med. 2012;366:1696-704.

12. Rodes-Cabau J, Webb JG, Cheung A, Ye J, Dumont E, Feindel CM, et al. Transcatheter aortic valve implantation for the treatment of severe symptomatic aortic stenosis in patients at very high or prohibitive surgical risk: acute and late outcomes of the multicenter Canadian experience. J Am Coll Cardiol. 2010;55: 1080-90.

13. Walther T, Rastan A, Falk V, Lehmann S, Garbade J, Funkat AK, et al. Patient prosthesis mismatch affects short- and long-term outcomes after aortic valve replacement. Eur J Cardiothorac Surg. 2006;30:15-9.

14. Awad W, Mathur A, Baldock L, Oliver S, Kennon S. Comparing post-operative resource consumption following transcatheter aortic valve implantation (TAVI) and conventional aortic valve replacement in the UK. J Med Econ. 2014;17: 357-64.

15. Hannan EL, Samadashvili Z, Jordan D, Sundt TM III, Stamato NJ, Lahey SJ, et al. Thirty-day readmissions after transcatheter aortic valve implantation versus surgical aortic valve replacement in patients with severe aortic stenosis in New York State. Circ Cardiovasc Interv. 2015; 8:e002744.

Key Words: aortic valve replacement, cardiac surgery, endovascular procedures, heart valve, transcatheter 